\title{
The Brenner-Hochster-Kollár and Whitney problems for vector-valued functions and jets
}

\author{
Charles Fefferman and Garving K. Luli
}

\begin{abstract}
In this paper, we give analytic methods for finding $C^{m}\left(\mathbb{R}^{n}\right)$ and $\mathrm{C}^{\mathrm{m}, 1}\left(\mathbb{R}^{n}\right)$ solutions of a finite system of linear equations. Along the way, we also solve a generalized Whitney problem for vector-valued functions and jets.
\end{abstract}

\section{Introduction}

In [13], the first author and J. Kollár studied the following problem:

Problem 1 (The Brenner-Hochster-Kollár problem). Let $\mathrm{X}$ be a space of continuous functions on a topological space $\mathrm{E}$. Suppose we are given $\mathbb{R}^{\mathrm{s}}$-valued functions $\mathrm{f}_{1}, \ldots, \mathrm{f}_{\mathrm{d}}$, and $\phi$ on $\mathrm{E}$. How can we decide whether there exist $\phi_{1}, \ldots, \phi_{\mathrm{d}} \in \mathrm{X}$ such that

$$
\sum_{i=1}^{\mathrm{d}} \phi_{\mathrm{i}} \mathrm{f}_{\mathrm{i}}=\phi \text { on } \mathrm{E} \text { ? }
$$

For $X=C^{0}(E)$ with $E=\mathbb{R}^{n},[13]$ gives two effective methods (one analytic and the other algebraic) for solving this problem when the given functions $f_{1}, \ldots, f_{d}$ and $\phi$ are polynomials. ([13] also treats more general E.) Problem 1 in that case arose from algebraic geometry; see Brenner [3], Epstein-Hochster [8], and Kollár [15]. When $\phi_{1}, \ldots, \phi_{\mathrm{d}}$ exist, the algebraic method in [13] produces semialgebraic $\phi_{1}, \ldots, \phi_{\mathrm{d}}$. On the other hand, the analytic method solves Problem 1 for $X=C^{0}\left(\mathbb{R}^{n}\right)$ without assuming that $f_{1}, \ldots, f_{d}$ and $\phi$ are polynomials. Here, we extend the analytic method in [13] to solve Problem 1 for $X=C^{m}\left(\mathbb{R}^{n}\right)$ (the space of real-valued functions whose derivatives up to order $m$ are continuous and bounded on $\mathbb{R}^{n}$ ) and $C^{m, \omega}\left(\mathbb{R}^{n}\right)$ (the space of real-valued functions whose $\mathrm{m}$-th derivatives have modulus of continuity $\omega$; see Section 2 for more details). These cases were left open in [13].

Mathematics Subject Classification (2010): Primary 46S30; Secondary 14Q99.

Keywords: Generalized Whitney problems, extending vector-valued functions, continuous closures, differentiable closures, Brenner-Hochster-Kollár problem. 
Our work on Problem 1 relates to:

Problem 2 (Whitney's extension problem). Let $\mathrm{X}$ denote a function space. Suppose we are given a compact set $\mathrm{E} \subset \mathbb{R}^{\mathrm{n}}$ and a function $\mathrm{f}: \mathrm{E} \rightarrow \mathbb{R}$. How can we decide whether there exists $\mathrm{F} \in \mathrm{X}$ such that $\mathrm{F}=\mathrm{f}$ on $\mathrm{E}$ ?

For $X=C^{m, \omega}\left(\mathbb{R}^{n}\right)$ and $X=C^{m}\left(\mathbb{R}^{n}\right)$, Problem 2 was solved in [11], [12], building on previous work of H. Whitney [20], [21], Brudnyi-Shvartsman [4], [5], [6], [7], and Bierstone-Milman-Pawłucki [2].

For $X=C^{m}\left(\mathbb{R}^{n}\right)$, we will solve a more general problem that includes both the Brenner-Hochster-Kollár and the Whitney problems as special cases. We believe that this general problem is of independent interest.

To facilitate the statement of our generalization of Problems 1 and 2, we introduce a few definitions and a bit of notation.

Let $C^{m}\left(\mathbb{R}^{n}, \mathbb{R}^{\mathrm{d}}\right)$ be the space of $\mathbb{R}^{\mathrm{d}}$-valued functions whose derivatives up to order $m$ are continuous and bounded on $\mathbb{R}^{n}$. We write $\mathcal{P}_{m, n}$ to denote the vector space of all (real) polynomials of degree at most $m$ on $\mathbb{R}^{n}$. For real-valued functions $F, J_{\chi}^{m} F$ stands for the $m$-jet at $x$, which we identify with the Taylor polynomial

$$
\widehat{x} \longmapsto \sum_{|\alpha| \leq m} \frac{1}{\alpha !}\left(\partial^{\alpha} F\right)(x)(\widehat{x}-x)^{\alpha} .
$$

Thus, the ring of $\mathcal{R}_{\mathrm{m}, \mathrm{n}}^{\mathrm{x}}$ of $\mathrm{m}$-jets of functions at $x$ is identified with $\mathcal{P}_{\mathrm{m}, \mathrm{n}}$, the space of real m-th degree polynomials on $\mathbb{R}^{n}$; and the multiplication $\odot_{m, n}^{x}$ in $\mathcal{R}_{\mathrm{m}, \mathrm{n}}^{\mathrm{x}}$ may be regarded as a multiplication on $\mathcal{P}_{\mathrm{m}, \mathrm{n}}$. Here, the multiplication $\odot_{m, n}^{x}$ is defined as $\mathrm{P} \odot_{\mathrm{m}, \mathrm{n}}^{\mathrm{x}} \mathrm{Q} \equiv \mathrm{J}_{\mathrm{x}}^{\mathrm{m}}(\mathrm{PQ})$ for $\mathrm{P}, \mathrm{Q} \in \mathcal{R}_{\mathrm{m}, \mathrm{n}}^{\mathrm{x}}$. For vector-valued functions $\vec{F}=\left(F_{1}, \ldots, F_{d}\right)$, we write $J_{x}^{m} \vec{F}$ to denote the vector

$$
\left(J_{x}^{m} F_{1}, \ldots, J_{x}^{m} F_{d}\right) \in \underbrace{\mathcal{P}_{m, n} \oplus \ldots \oplus \mathcal{P}_{m, n}}_{d}=\left(\mathcal{P}_{m, n}\right)^{d} .
$$

We regard $\left(\mathcal{P}_{\mathrm{m}, \mathrm{n}}\right)^{\mathrm{d}}$ as an $\mathcal{R}_{\mathrm{m}, \mathrm{n}}^{\mathrm{x}}$-module by the multiplication rule

$$
\mathrm{Q} \odot_{m, n}^{x}\left(P_{1}, \ldots, P_{d}\right)=\left(Q \odot_{m, n}^{x} P_{1}, \ldots, Q \odot_{m, n}^{x} P_{d}\right) .
$$

To motivate the next few definitions, we note that the $m$-jet $\left(P_{1}, \ldots, P_{d}\right)$ at $x$ of any solution of (1.1) belongs to

$$
\begin{aligned}
\vec{H}(x) & =\left\{\vec{P}=\left(P_{1}, \ldots, P_{d}\right): P_{1}(x) f_{1}(x)+\ldots+P_{d}(x) f_{d}(x)=\phi(x)\right\} \\
& \subset\left(\mathcal{P}_{m, n}\right)^{d} .
\end{aligned}
$$

Here, the affine subspace $\vec{H}(x) \subset\left(\mathcal{P}_{m, n}\right)^{d}$ may be computed from (1.1) by elementary linear algebra. Perhaps $\vec{H}(x)$ is empty. (By convention, we allow the empty set, single points, and all of $\left(\mathcal{P}_{m, n}\right)^{d}$ as affine subspaces of $\left(\mathcal{P}_{m, n}\right)^{d}$.) If $\vec{H}(x)$ is nonempty and if $\vec{P}_{x}^{0}$ is any element of $\vec{H}(x)$, then we may express

$$
\overrightarrow{\mathrm{H}}(x)=\overrightarrow{\mathrm{P}}_{x}^{0}+\overrightarrow{\mathrm{I}}(x),
$$

where $\vec{I}(x)=\left\{\vec{P}=\left(P_{1}, \ldots, P_{d}\right): P_{1}(x) f_{1}(x)+\cdots+P_{d}(x) f_{d}(x)=0\right\}$ is an $\mathcal{R}_{m, n^{-}}^{x}$ submodule of the $\mathcal{R}_{\mathrm{m}, \mathrm{n}}^{\mathrm{x}}$-module $\left(\mathcal{P}_{\mathrm{m}, \mathrm{n}}\right)^{\mathrm{d}}$. 
A vector of functions $\vec{F} \in C^{m}\left(\mathbb{R}^{n}, \mathbb{R}^{d}\right)$ solves equation (1.1) if and only if $\mathrm{J}_{x}^{\mathrm{m}} \overrightarrow{\mathrm{F}} \in \overrightarrow{\mathrm{H}}(x)$ for all $x \in \mathbb{R}^{n}$.

Similarly, let $f: E \rightarrow \mathbb{R}$ be as in Problem 2. For $x \in E$ define

$$
H(x)=\left\{P \in \mathcal{P}_{m, n}: P(x)=f(x)\right\},
$$

where

$$
f^{x}=\text { the constant polynomial } \widehat{x} \longmapsto f(x) \text {, }
$$

and

$$
\mathrm{I}(\mathrm{x})=\left\{\mathrm{P} \in \mathcal{P}_{\mathrm{m}, \mathrm{n}}: \mathrm{P}(\mathrm{x})=0\right\} .
$$

Then $H(x)=f^{x}+I(x), I(x)$ is an ideal in $\mathcal{R}_{m, n}^{x}$, and a function $F \in C^{m}\left(\mathbb{R}^{n}, \mathbb{R}\right)$ satisfies $F=f$ on $E$ if and only if $J_{x}^{m} F \in H(x)$ for all $x \in E$.

The above remarks motivate the following definitions. Fix integers $m \geq 0, n \geq 1$, and $d \geq 1$. Let $E \subset \mathbb{R}^{n}$ be compact. A bundle over $E$ is a family $\overrightarrow{\mathcal{H}}=(\overrightarrow{\mathrm{H}}(x))_{x \in E}$ of (possibly empty) affine subspaces $\vec{H}(x) \subset\left(\mathcal{P}_{m, n}\right)^{d}$, parametrized by the points $x \in E$, such that each nonempty $\vec{H}(x)$ has the form

$$
\overrightarrow{\mathrm{H}}(x)=\overrightarrow{\mathrm{P}}^{x}+\overrightarrow{\mathrm{I}}(x)
$$

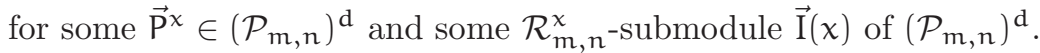

We make no assumptions as to how $\overrightarrow{\mathrm{H}}(x), \overrightarrow{\mathrm{P}}^{x}$, and $\overrightarrow{\mathrm{I}}(x)$ depend on $x$.

We call $\vec{H}(x)$ the fiber of $\overrightarrow{\mathcal{H}}$ at $x$. If $\overrightarrow{\mathcal{H}}^{\prime}=\left(\vec{H}^{\prime}(x)\right)_{x \in E}$ is another bundle over $E$, then we call $\overrightarrow{\mathcal{H}}^{\prime}$ a subbundle of $\overrightarrow{\mathcal{H}}$ provided $\overrightarrow{\mathrm{H}}^{\prime}(x) \subseteq \overrightarrow{\mathrm{H}}(x)$ for each $x \in \mathrm{E}$. If $\overrightarrow{\mathcal{H}}^{\prime}$ is a subbundle of $\overrightarrow{\mathcal{H}}$, then we write $\overrightarrow{\mathcal{H}} \supseteq \overrightarrow{\mathcal{H}}^{\prime}$. Finally, a section of a bundle $\overrightarrow{\mathcal{H}}=$ $(\overrightarrow{\mathrm{H}}(x))_{x \in E}$ is an $\mathbb{R}^{\mathrm{d}}$-valued function $\overrightarrow{\mathrm{F}} \in \mathrm{C}^{\mathrm{m}}\left(\mathbb{R}^{n}, \mathbb{R}^{\mathrm{d}}\right)$ such that $J_{x}^{m} \overrightarrow{\mathrm{F}} \in \overrightarrow{\mathrm{H}}(x)$ for each $x \in \mathrm{E}$.

We can now state:

Problem 3 (Generalized Whitney's extension problem for $\mathrm{C}^{\mathrm{m}}$ ). Fix $\mathrm{m}, \mathrm{n}$ and $\mathrm{d}$. How can we decide whether a given bundle $\overrightarrow{\mathcal{H}}=(\overrightarrow{\mathrm{H}}(\mathrm{x}))_{\mathrm{x} \in \mathrm{E}}$ has a section?

From our discussion of the bundles formed by (1.2) and by (1.3), we see that Problem 1 for $X=C^{m}\left(\mathbb{R}^{n}, \mathbb{R}^{d}\right)$ and Problem 2 for $X=C^{m}\left(\mathbb{R}^{n}, \mathbb{R}\right)$ are special cases of Problem 3.

For the scalar case (i.e., $\mathrm{d}=1$ ), Problem 3 is well understood thanks to Bierstone-Milman-Pawłucki [2] and the first author [12] (see references therein). Problem 3 for $X=C^{0}\left(\mathbb{R}^{n}, \mathbb{R}^{d}\right)$ is solved in [13]. In this paper, we solve Problem 3 for all $m n$ and $d$ by reducing it to the known scalar case $d=1$.

A variant of Problem 3 with $C^{m}\left(\mathbb{R}^{n}, \mathbb{R}^{d}\right)$ replaced by $C^{m, \omega}\left(\mathbb{R}^{n}, \mathbb{R}^{d}\right)$ is also of interest. Here, $C^{m, \omega}\left(\mathbb{R}^{n}, \mathbb{R}^{d}\right)$ is the space of $C^{m}$ functions whose $m$-th derivatives have a given modulus of continuity $\omega$ (see Section 2). More precisely, we assume that $\omega$ is a "regular modulus of continuity" (again, see Section 2). 
Problem 4 (Generalized Whitney's extension problem for $\mathrm{C}^{\mathrm{m}, \omega}$ ). Fix $\mathrm{m}$, n and $\mathrm{d}$. Let $\mathrm{E}$ be an (arbitrary) given subset of $\mathbb{R}^{\mathrm{n}}$. Suppose at each $\mathrm{x} \in \mathrm{E}$, we are given an $m$-jet $\overrightarrow{\mathrm{f}}(\mathrm{x}) \in\left(\mathcal{P}_{\mathrm{m}, \mathrm{n}}\right)^{\mathrm{d}}$ and a convex set $\sigma(\mathrm{x}) \subseteq\left(\mathcal{P}_{\mathrm{m}, \mathrm{n}}\right)^{\mathrm{d}}$, with $\sigma(x)$ symmetric about the origin. How can decide whether there exist $\overrightarrow{\mathrm{F}} \in \mathrm{C}^{\mathrm{m}, \omega}\left(\mathbb{R}^{\mathrm{n}}, \mathbb{R}^{\mathrm{d}}\right)$ and $\mathrm{M}<\infty$ such that $\mathrm{J}_{\mathrm{x}}^{\mathrm{m}} \overrightarrow{\mathrm{F}}-\overrightarrow{\mathrm{f}}(\mathrm{x}) \in \mathrm{M \sigma}(\mathrm{x})$ for all $\mathrm{x} \in \mathrm{E}$ ?

For the case $d=1$, Problem 4 has been extensively studied (see [4], [5], [6], [7], [9], [10] and [11]). More specifically, for $d=1$, if the convex sets $\sigma(x)$ are assumed to satisfy a condition called "Whitney $\omega$-convexity," then a complete answer to Problem 4 is given in [9].

In this paper, we formulate the notion of Whitney $\omega$-convexity for the general case (all $\mathrm{m}, \mathrm{n}$ and $\mathrm{d}$ ), and solve Problem 4 under the assumption that the convex sets $\sigma(x)$ are Whitney $\omega$-convex.

We will see that every $\mathcal{R}_{m, n}^{x}$-submodule of $\left(\mathcal{P}_{m, n}\right)^{d}$ is Whitney $\omega$-convex. Consequently, Problem 4 includes the direct analogue of Problem 3 with $C^{m}\left(\mathbb{R}^{n}, \mathbb{R}^{d}\right)$ replaced by $C^{\mathrm{m}, \omega}\left(\mathbb{R}^{\mathrm{n}}, \mathbb{R}^{\mathrm{d}}\right)$. Thus, by solving Problems 3 and 4 as promised above, we also solve Problem 1 for $X=C^{m}\left(\mathbb{R}^{n}, \mathbb{R}^{d}\right)$ and for $X=C^{m, \omega}\left(\mathbb{R}^{n}, \mathbb{R}^{d}\right)$.

Our definition of Whitney $\omega$-convexity is given in Section 2. As we explain there, each Whitney $\omega$-convex set has a "Whitney constant" $A \geq 1$. In a spirit similar to that of [9], we solve Problem 4 for Whitney $\omega$-convex $\sigma(x)$ by means of the following:

Theorem 1. Fix $\mathrm{m}, \mathrm{n}$ and $\mathrm{d}$. Then there exists an integer $\mathrm{k}^{\#}$ (depending only on the integers $\mathrm{m}, \mathrm{n}$ and $\mathrm{d}$ ) such that the following holds. Let $\mathrm{E} \subset \mathbb{R}^{\mathrm{n}}$ be an arbitrary subset and let $\omega$ be a regular modulus of continuity. Suppose for each $x \in \mathrm{E}$, we are given an $\mathrm{m}$-jet $\overrightarrow{\mathrm{f}}(\mathrm{x}) \in\left(\mathcal{P}_{\mathrm{m}, \mathrm{n}}\right)^{\mathrm{d}}$ and a Whitney $\omega$-convex set $\sigma(\mathrm{x}) \subseteq\left(\mathcal{P}_{\mathrm{m}, \mathrm{n}}\right)^{\mathrm{d}}$ (with Whitney constant $\mathrm{A})$. Let $\mathrm{M}$ be a positive real number.

Assume the following condition is satisfied: for each $\mathrm{S} \subseteq \mathrm{E}$ with \# $(\mathrm{S}) \leq \mathrm{k}^{\#}$, there exists $\overrightarrow{\mathrm{F}}^{\mathrm{S}} \in \mathrm{C}^{\mathrm{m}, \omega}\left(\mathbb{R}^{\mathrm{n}}, \mathbb{R}^{\mathrm{d}}\right)$ such that

(i) $J_{x}^{m} \vec{F}^{S} \in \vec{f}(x)+M \sigma(x)$ for all $x \in S$;

(ii) $\|\overrightarrow{\mathrm{F}} \mathrm{S}\|_{\mathrm{C}^{\mathrm{m}, \omega}\left(\mathbb{R}^{\mathrm{n}}, \mathbb{R}^{\mathrm{d}}\right)} \leq \mathrm{M}$.

Then there exists $\vec{F} \in C^{m, \omega}\left(\mathbb{R}^{n}, \mathbb{R}^{d}\right)$ such that $J_{x}^{m} \vec{F} \in \vec{f}(x)+C(A) M \sigma(x)$ for all $\mathrm{x} \in \mathrm{E}$, and $\|\overrightarrow{\mathrm{F}}\|_{\mathrm{C}^{\mathrm{m}, \omega}\left(\mathbb{R}^{n}, \mathbb{R}^{\mathrm{d}}\right)} \leq \mathrm{C}(\mathrm{A}) \mathrm{M}$. Here, $\mathrm{C}(\mathrm{A})$ depends only on $\mathrm{A}, \mathrm{m}, \mathrm{n}, \mathrm{d}$.

Theorem 1 is a type of "Finiteness Principle"; the constant $k^{\#}$ is often referred to as a "finiteness constant." The idea of the Finiteness Principle originated in the work of Brudnyi-Shvartsman (see [5] and [16]). In essence, Theorem 1 reduces Problem 4 for a general set $E$ to the special case of finite $E$ with bounded cardinality. This special case is readily solvable, as we explain in Section 5.

We are pleasantly surprised to learn that the proof of Theorem 1 follows from the scalar case $(d=1)$, which has been proven in [9]. We should also remark that P. Shvartsman has communicated to us his unpublished proof of Theorem 1 for the case $\mathrm{m}=0$, as a consequence of his results [17], [18] on "Lipschitz selection." 
To explain our solution to Problem 3, we need to introduce some more terminology.

Fix $\mathrm{m}, \mathrm{n}$ and $\mathrm{d}$, and let $\mathrm{k}^{\#}$ be a large enough constant depending only on $\mathrm{m}, \mathrm{n}$ and $d$ (see Section 4 for a discussion on the size of $k^{\#}$ ).

Let $\overrightarrow{\mathcal{H}}=(\overrightarrow{\mathrm{H}}(x))_{x \in \mathrm{E}}$ be a bundle. Then the "Glaeser refinement" $\overrightarrow{\mathcal{H}}^{\prime}=\left(\overrightarrow{\mathrm{H}}^{\prime}(x)\right)_{x \in \mathrm{E}}$ is a subbundle of $\overrightarrow{\mathcal{H}}$, defined as follows.

Given $x_{0} \in E$ and $\vec{P}_{0} \in \vec{H}\left(x_{0}\right)$, we say that $\vec{P}_{0} \in \vec{H}^{\prime}\left(x_{0}\right)$ if and only if the following condition holds: given $\varepsilon>0$, there exists $\delta>0$ such that for all $x_{1}, \ldots, x_{k \#} \in E \cap B\left(x_{0}, \delta\right)$, there exist $\vec{P}_{1} \in \vec{H}\left(x_{1}\right), \ldots, \vec{P}_{k^{\#}} \in \vec{H}\left(x_{k^{\#}}\right)$ with

$$
\left|\partial^{\alpha}\left(\vec{P}_{i}-\vec{P}_{j}\right)\left(x_{i}\right)\right| \leq \varepsilon\left|x_{i}-x_{j}\right|^{m-|\alpha|},
$$

for $|\alpha| \leq m$ and $0 \leq i, j \leq k^{\#}$. (Compare with Glaeser [14], Bierstone-MilmanPawłucki [2], C. Fefferman [12], and C. Fefferman-Kollár [13].)

The Glaeser refinement has three crucial properties:

(P1) $\overrightarrow{\mathcal{H}}^{\prime}$ is a subbundle of $\overrightarrow{\mathcal{H}}$.

(P2) Any section of $\overrightarrow{\mathcal{H}}$ is also a section of $\overrightarrow{\mathcal{H}}^{\prime}$. (This follows easily from Taylor's theorem.)

(P3) In principle, $\overrightarrow{\mathcal{H}}^{\prime}$ may be computed from $\overrightarrow{\mathcal{H}}$ by doing elementary linear algebra and computing a limsup. We explain this in Section 5 below.

Let us consider the implication of the Glaeser refinement for Problem 3. Starting with a bundle $\overrightarrow{\mathcal{H}}_{0}$ over $E$ and repeatedly taking the Glaeser refinement, we obtain a sequence of bundles

$$
\overrightarrow{\mathcal{H}}_{0} \supseteq \overrightarrow{\mathcal{H}}_{1} \supseteq \overrightarrow{\mathcal{H}}_{2} \supseteq \cdots \quad \text { over E. }
$$

For each $l, \overrightarrow{\mathcal{H}}_{l+1}$ is the Glaeser refinement of $\overrightarrow{\mathcal{H}}_{l}$. By (P1) and (P2), the bundles $\overrightarrow{\mathcal{H}}_{l}$ have the same sections. Therefore, Problem 3 for a given bundle $\overrightarrow{\mathcal{H}}_{0}$ is the same as Problem 3 for any of the iterated Glaeser refinements $\overrightarrow{\mathcal{H}}_{l}$.

A lemma adapted from [12] (which in turn is adapted from [14] and [2]) shows that $\overrightarrow{\mathcal{H}}_{l}=\left(\overrightarrow{\mathrm{H}}_{\mathrm{l}}(\mathrm{x})\right)_{\mathrm{x} \in \mathrm{E}}$ stablizes after a finite number of iterations. More precisely, for $L=2 \operatorname{dim}\left[\left(\mathcal{P}_{m, n}\right)^{d}\right]+1$, we have $\vec{H}_{l}(x)=\vec{H}_{L}(x)$ for all $l \geq L$ and $x \in E$. For the sake of completeness, we reproduce a proof of this in Section 5 below.

Passing from $\overrightarrow{\mathcal{H}}_{0}$ to $\overrightarrow{\mathcal{H}}_{\mathrm{L}}$, we therefore reduce Problem 3 to the special case in which $\overrightarrow{\mathcal{H}}$ is its own Glaeser refinement.

This special case of Problem 3 is solved by means of the following.

Theorem 2. Let $\overrightarrow{\mathcal{H}}=(\overrightarrow{\mathrm{H}}(\mathrm{x}))_{\mathrm{x} \in \mathrm{E}}$ be a bundle. Suppose $\overrightarrow{\mathcal{H}}$ is its own Glaeser refinement and each fiber of $\overrightarrow{\mathcal{H}}$ is nonempty. Then $\overrightarrow{\mathcal{H}}$ admits a section.

In the scalar case $d=1$, Theorem 2 is proven in [12]. We will prove Theorem 2 in general by reducing it to the known scalar case, just as for Theorem 1.

We remark that our methods for solving Problem 1 for $X=C^{m, \omega}(E)$ and $X=C^{m}(E)$ with $E=\mathbb{R}^{n}$ apply equally well to the solution of Problem 1 for $X=C^{m, \omega}(E)$ and $X=C^{m}(E)$ with $E$ being a manifold. 
Acknowledgements. We are grateful to Arie Israel, Bo'az Klartag, János Kollár, Assaf Naor, and Pavel Shvartsman for valuable discussions. We are grateful also to the American Institute of Mathematics (AIM) and the Office of Naval Research (ONR) for supporting workshops at which some of those conversations occurred. We thank János Kollár for helpful suggestions that led to an improvement of the manuscript. Part of the research was conducted while the second author was visiting the Mathematical Sciences Center at Tsinghua University, China; the center's hospitality is gratefully acknowledged.

\section{Notation and definitions}

We fix integers $d \geq 1, n \geq 1$ and $m \geq 0$. $C^{m}\left(\mathbb{R}^{n}, \mathbb{R}^{d}\right)$ denotes the space of functions $F: \mathbb{R}^{n} \rightarrow \mathbb{R}^{d}$ whose derivatives up to order $m$ are continuous and bounded on $\mathbb{R}^{n}$. For $\vec{F}=\left(F_{1}, \ldots, F_{d}\right) \in C^{m}\left(\mathbb{R}^{n}, \mathbb{R}^{d}\right)$, we define the norm

$$
\|\vec{F}\|_{C^{m}\left(\mathbb{R}^{n}, \mathbb{R}^{d}\right)}=\sup _{x \in \mathbb{R}^{n}} \max _{1 \leq j \leq d,|\alpha| \leq m}\left|\partial^{\alpha} F_{j}(x)\right| .
$$

$C^{m, \omega}\left(\mathbb{R}^{n}, \mathbb{R}^{d}\right)$ denotes the space of all $C^{m}\left(\mathbb{R}^{n}, \mathbb{R}^{d}\right)$ functions $\vec{F}: \mathbb{R}^{n} \rightarrow \mathbb{R}^{d}$ for which the norm

$$
\begin{aligned}
& \|\overrightarrow{\mathrm{F}}\|_{C^{m}, \omega\left(\mathbb{R}^{n}, \mathbb{R}^{\mathrm{d}}\right)} \\
& \quad=\max \left\{\|\overrightarrow{\mathrm{F}}\|_{C^{m}\left(\mathbb{R}^{n}, \mathbb{R}^{\mathrm{d}}\right),} \sup _{x, y \in \mathbb{R}^{n}, 0<|x-y| \leq 1} \max _{1 \leq j \leq d,|\alpha|=m}\left|\frac{\partial^{\alpha} F_{j}(x)-\partial^{\alpha} F_{j}(y)}{\omega(|x-y|)}\right|\right\}
\end{aligned}
$$

is finite.

A function $\omega:[0,1] \rightarrow[0, \infty)$ is called a "regular modulus of continuity" if it satisfies the following conditions:

- $\omega(0)=\lim _{t \rightarrow 0+} \omega(t)=0$ and $\omega(1)=1$.

- $\omega(t)$ is increasing on $[0,1]$.

- $\omega(t) / t$ is decreasing on $(0,1]$.

Fix $x \in \mathbb{R}^{n}$. We say that $\sigma(x) \subseteq\left(\mathcal{P}_{m, n}\right)^{d}$ is "Whitney $\omega$-convex (in $\left.\left(\mathcal{P}_{m, n}\right)^{\mathrm{d}}\right)$ at $x$ with Whitney constant $A$ " if the following conditions are satisfied:

- $\sigma(x)$ is closed, convex, symmetric (that is, $\vec{P} \in \sigma(x)$ if and only if $-\vec{P} \in \sigma(x)$ ).

- Let $\left(P_{1}, \ldots, P_{d}\right) \in \sigma(x), Q \in \mathcal{R}_{m, n}^{x}$, and $\delta \in(0,1]$. Assume $\left(P_{1}, \ldots, P_{d}\right)$ and $\mathrm{Q}$ satisfy the following estimates:

$$
\begin{aligned}
& \left|\partial^{\alpha} P_{j}(x)\right| \leq \omega(\delta) \delta^{m-|\alpha|} \text { for } 1 \leq j \leq d \text { and }|\alpha| \leq m ; \\
& \left|\partial^{\alpha} Q(x)\right| \leq \delta^{-|\alpha|} \text { for }|\alpha| \leq m .
\end{aligned}
$$

Then

$$
\left(\mathrm{P}_{1} \odot_{\mathrm{m}, \mathrm{n}}^{\mathrm{x}} \mathrm{Q}, \ldots, \mathrm{P}_{\mathrm{d}} \odot_{\mathrm{m}, \mathrm{n}}^{\mathrm{x}} \mathrm{Q}\right) \in A \sigma(x),
$$

where $\odot_{\mathrm{m}, \mathrm{n}}^{\mathrm{x}}$ denotes the multiplication in $\mathcal{R}_{\mathrm{m}, \mathrm{n}}^{\mathrm{x}}$.

From the definition of Whitney $\omega$-convexity, it immediately follows that every $\mathcal{R}_{\mathrm{m}, \mathrm{n}}^{\mathrm{x}}$-submodule of $\left(\mathcal{P}_{\mathrm{m}, \mathrm{n}}\right)^{\mathrm{d}}$ is Whitney $\boldsymbol{\omega}$-convex with Whitney constant 1. 


\section{Finiteness principle for $\mathrm{C}^{\mathrm{m}, \omega}$}

In this section, we prove Theorem 1 .

We suppose we are given an arbitrary subset $E \subset \mathbb{R}^{n}$, a regular modulus of continuity $\omega$, a vector-valued m-jet $\vec{f}(x) \in\left(\mathcal{P}_{m, n}^{x}\right)^{d}$ and a Whitney $\omega$-convex set $\sigma(x) \subseteq\left(\mathcal{P}_{\mathrm{m}, \mathrm{n}}\right)^{\mathrm{d}}$ (with Whitney constant $A$ ) at each point $x \in \mathrm{E}$.

We denote by $\widehat{x}=\left(\widehat{x}_{1}, \ldots, \widehat{x}_{n}\right)$ a dummy variable in $\mathbb{R}^{n}$ and $\widehat{v}=\left(\widehat{v}_{1}, \ldots, \widehat{v}_{d}\right)$ a dummy variable in $\mathbb{R}^{\mathrm{d}}$.

We note that if $\mathrm{P}(\widehat{x}, \widehat{v})$ is an $(m+1)$-jet on $\mathbb{R}^{n+d}$, then $\left.[\mathrm{P}(\widehat{x}, \widehat{v})]\right|_{\widehat{v}=0}$ is an $(m+1)$-jet on $\mathbb{R}^{n}$, and $\left.\left[\partial_{\widehat{v}_{j}} \mathrm{P}\right]\right|_{\widehat{v}=0}$ is an $m$-jet on $\mathbb{R}^{n}$.

To prove the theorem, we will show that there exists $G(\widehat{x}, \widehat{v}): \mathbb{R}^{n+d} \rightarrow \mathbb{R}$ such that

1. $\mathrm{G}(\widehat{x}, 0) \equiv 0$;

2. $\left(J_{x}^{m}\left[\left.\partial_{\widehat{v}_{1}} G(\widehat{x}, \widehat{v})\right|_{\widehat{v}=0}\right], \ldots, J_{x}^{m}\left[\left.\partial_{\widehat{v}_{d}} G(\widehat{x}, \widehat{v})\right|_{\widehat{v}=0}\right]\right) \in \vec{f}(x)+\operatorname{MC} \sigma(x)$ for all $x \in E$

3. $\|\mathrm{G}\|_{\mathrm{C}^{\mathrm{m}+1, \omega}\left(\mathbb{R}^{n+\mathrm{d}}\right)} \leq \mathrm{C} M$, for some constant $\mathrm{C}$ depending only on $\mathrm{m}, \mathrm{n}$, and $d$.

Once this is proven, the theorem follows at once by taking

$$
\overrightarrow{\mathrm{F}}(\widehat{x})=\left(\left.\left[\partial_{\widehat{v}_{1}} \mathrm{G}(\widehat{x}, \widehat{v})\right]\right|_{\widehat{v}=0}, \ldots,\left.\left[\partial_{\widehat{v}_{\mathrm{d}}} \mathrm{G}(\widehat{x}, \widehat{v})\right]\right|_{\widehat{v}=0}\right) .
$$

First, we recall the following result (Theorem 3 in [9]); our proof of Theorem 1 will be based on it.

Theorem 3 (Finiteness principle for real-valued jets, C. Fefferman [9]). Given integers $\mathrm{m} \geq 0$ and $\mathrm{n} \geq 1$, there exists $\mathrm{k}^{\#}$, depending only on $\mathrm{m}$ and $\mathrm{n}$, such that the following holds.

Let $\omega$ be a regular modulus of continuity, $\overline{\mathrm{E}} \subset \mathbb{R}^{\mathrm{n}}$ an arbitrary subset, and $\overline{\mathrm{A}}>0$. Suppose for each $\overline{\mathrm{x}} \in \overline{\mathrm{E}}$, we are given a (real-valued) $\mathrm{m}$-jet $\overline{\mathrm{f}}(\mathrm{x}) \in \mathcal{R}_{\mathrm{m}, \mathrm{n}}^{\mathrm{x}}$, and a Whitney $\omega$-convex set $\bar{\sigma}(x) \subseteq \mathcal{R}_{\mathrm{m}, \mathrm{n}}^{\mathrm{x}}$ with Whitney constant $\overline{\mathrm{A}}$.

Assume the following condition is satisfied: For each $\mathrm{S} \subseteq \overline{\mathrm{E}}$ with $\#(\mathrm{~S}) \leq \mathrm{k}^{\#}$, there exists a map $\mathrm{x} \longmapsto \mathrm{P}^{\mathrm{x}}$ from $\mathrm{S} \rightarrow \mathcal{P}_{\mathrm{m}, \mathrm{n}}$ such that $(\mathrm{i}) \mathrm{P}^{\mathrm{x}} \in \overline{\mathrm{f}}(\mathrm{x})+\mathrm{M} \bar{\sigma}(\mathrm{x})$ for all $x \in S$; (ii) $\left|\partial^{\beta} \mathrm{P}^{\mathrm{x}}(\mathrm{x})\right| \leq \mathrm{M}$ for all $\mathrm{x} \in \mathrm{S},|\beta| \leq \mathrm{m}$; and (iii) $\left|\partial^{\beta}\left(\mathrm{P}^{\mathrm{x}}-\mathrm{P}^{\mathrm{y}}\right)(\mathrm{x})\right| \leq$ $\omega(|x-y|)|x-y|^{m-|\beta|}$ for $|\beta| \leq m$ and $|x-y| \leq 1, x, y \in S$.

Then there exists $\mathrm{G} \in \mathrm{C}^{\mathrm{m}, \omega}\left(\mathbb{R}^{\mathrm{n}}\right)$ such that

(i) $\|\mathrm{G}\|_{\mathrm{C}^{\mathrm{m}, \omega}\left(\mathbb{R}^{\mathrm{n}}\right)} \leq \mathrm{C} \mathrm{M}$ and

(ii) $J_{x}^{m} G \in \bar{f}(x)+C M \bar{\sigma}(x)$ for all $x \in E$.

Here, $\mathrm{C}$ depends only on $\overline{\mathrm{A}}, \mathrm{m}$, and $\mathrm{n}$.

Remark 1. In [7], Brudnyi-Shvartsman showed Theorem 3 for $C^{1, \omega}\left(\mathbb{R}^{n}\right)$ and $\bar{\sigma}(x) \equiv 0$ (for all $x \in E$ ) with the sharp constant $k^{\#}=3 \times 2^{n-1}$. Theorem 3 was first conjectured by Brudnyi and Shvartsman. It was proven by C. Fefferman [9] with a large constant $k^{\#}$ (depending only on $m$ and $n$ ). Later, Bierstone-Milman 
in [1] and Shvartsman in [19] independently improved the constant $k^{\#}$ in the case $\bar{\sigma}(\chi) \equiv 0$ by showing that $k^{\#}=2^{\operatorname{dim}\left(\mathcal{P}_{m, n}\right)}$ is sufficient. For Whitney $\omega$ convex sets $\bar{\sigma}$, Shvartsman in [19] also showed that Theorem 3 holds with $\mathrm{k}^{\#}=$ $2^{\min \left\{l+1, \operatorname{dim} \mathcal{P}_{m, n}\right\}}$, where $l=\max _{x \in \overline{\mathrm{E}}} \operatorname{dim} \bar{\sigma}(x)$.

For each $x \in E$, define

$\widehat{\sigma}((x, 0))=\left\{\widehat{P} \in \mathcal{R}_{m+1, n+d}^{(x, 0)}:\left.\widehat{P}\right|_{\widehat{v}=0} \equiv 0\right.$ and $\left.\left(\left.\left[\partial_{\widehat{v}, ~} \widehat{P}\right]\right|_{\widehat{v}=0}, \ldots,\left.\left[\partial_{\widehat{v}_{d}} \widehat{P}\right]\right|_{\widehat{v}=0}\right) \in \sigma(x)\right\}$, where $\sigma(x) \subseteq\left(\mathcal{P}_{\mathrm{m}, \mathrm{n}}\right)^{\mathrm{d}}$ is as given in Theorem 1 .

Lemma 3.1. $\widehat{\sigma}((x, 0))$ is Whitney $\omega$-convex in $\mathcal{R}_{m+1, n+d}^{(x, 0)}$ with Whitney constant $A$.

Proof. That $\widehat{\sigma}((x, 0))$ is closed, convex, and symmetric follows directly from the definition of $\widehat{\sigma}((x, 0))$ and the fact that $\sigma(x)$ is Whitney $\omega$-convex.

Suppose $\widehat{P} \in \widehat{\sigma}((x, 0))$ and $\widehat{Q} \in \mathcal{R}_{m+1, n+d}^{(x, 0)}$.

Assume

$$
\left|\partial^{\gamma} \widehat{\mathrm{P}}(x, 0)\right| \leq \omega(\delta) \delta^{\mathrm{m}+1-|\gamma|}
$$

and

$$
\left|\partial^{\gamma} \widehat{Q}(x, 0)\right| \leq \delta^{-|\gamma|}
$$

for $|\gamma| \leq m+1$.

We need to show that $\widehat{P} \odot_{m+1, n+d}^{(x, 0)} \widehat{Q} \in A \widehat{\sigma}((x, 0))$, where $\odot_{m+1, n+d}^{(x, 0)}$ denotes the multiplication in $\mathcal{R}_{\mathrm{m}+1, \mathrm{n}+\mathrm{d}}^{(x, 0)}$.

First of all, we have

$$
\left.\left.\left.\left[\widehat{P} \odot_{m+1, n+d}^{(x, 0)} \widehat{Q}\right]\right|_{\widehat{v}=0} \equiv \widehat{P}\right|_{\widehat{v}=0} \odot_{m+1, n}^{x} \widehat{Q}\right|_{\widehat{v}=0} \equiv 0,
$$

since $\left.\widehat{\mathrm{P}}\right|_{\widehat{v}=0} \equiv 0$ by virtue of the fact that $\widehat{\mathrm{P}} \in \widehat{\sigma}((x, 0))$.

Let $\pi_{\mathrm{m}}: \mathcal{R}_{\mathrm{m}+1, \mathrm{n}+\mathrm{d}}^{(\mathrm{x}, 0)} \rightarrow \mathcal{R}_{\mathrm{m}, \mathrm{n}+\mathrm{d}}^{(\mathrm{x}, 0)}$ be the natural projection. We have

$$
\begin{aligned}
\left.\left(\partial_{\widehat{v}_{1}}\left[\widehat{P} \odot_{m+1, n+d}^{(x, 0)} \widehat{Q}\right], \ldots, \partial_{\widehat{v}_{d}}\left[\widehat{P} \odot_{m+1, n+d}^{(x, 0)} \widehat{Q}\right]\right)\right|_{\widehat{v}=0} & \\
= & \left(\left.\left.\partial_{\widehat{v}_{1}} \widehat{P}\right|_{\widehat{v}=0} \odot_{m, n}^{x} \pi_{m} \widehat{Q}\right|_{\widehat{v}=0}+\left.\left.\pi_{m} \widehat{P}\right|_{\widehat{v}=0} \odot_{m, n}^{x} \partial_{\widehat{v}, 1} \widehat{Q}\right|_{\widehat{v}=0}, \ldots,\right. \\
& \left.\left.\left.\partial_{\widehat{v}_{d}} \widehat{P}\right|_{\widehat{v}=0} \odot_{m, n}^{x} \pi_{m} \widehat{Q}\right|_{\widehat{v}=0}+\left.\left.\pi_{m} \widehat{P}\right|_{\widehat{v}=0} \odot_{m, n}^{x} \partial_{\widehat{v}} \widehat{Q}\right|_{\widehat{v}=0}\right) \\
= & \left(\left.\left.\partial_{\widehat{v}, 1} \widehat{P}\right|_{\widehat{v}=0} \odot_{m, n}^{x} \pi_{m} \widehat{Q}\right|_{\widehat{v}=0}, \ldots,\left.\left.\partial_{\widehat{v}} \widehat{P}\right|_{\widehat{v}=0} \odot_{m, n}^{x} \pi_{m} \widehat{Q}\right|_{\widehat{v}=0}\right), \\
& \text { since }\left.\widehat{P}\right|_{\widehat{v}=0} \equiv 0 .
\end{aligned}
$$

We write $\partial^{\gamma}=\partial_{\widehat{x}}^{\alpha} \partial_{\widehat{v}}^{\beta}$. If $\beta=1$, from (3.1), we have $\left|\partial_{\widehat{x}}^{\alpha} \partial_{\widehat{v}}^{\beta} \widehat{P}(x, 0)\right| \leq \omega(\delta) \delta^{m-|\alpha|}$. Therefore, we have

$$
\left|\partial_{\widehat{x}}^{\alpha}\left[\left.\left(\partial_{\widehat{v}} \widehat{P}\right)\right|_{\widehat{v}=0}\right](x)\right| \leq \omega(\delta) \delta^{m-|\alpha|} \text { for } 1 \leq j \leq d,|\alpha| \leq m .
$$

From (3.2), we have

$$
\left|\partial_{\widehat{x}}^{\alpha}\left[\left.\pi_{m} \widehat{Q}\right|_{\widehat{v}=0}\right](x)\right|=\left|\partial_{\widehat{x}}^{\alpha} \widehat{Q}(x, 0)\right| \leq \delta^{-|\alpha|} \text { for }|\alpha| \leq m .
$$


From (3.4), (3.5), (3.6), and the assumption that $\sigma(x)$ is Whitney $\omega$-convex (in $\left.\left(\mathcal{P}_{\mathrm{m}, \mathrm{n}}\right)^{\mathrm{d}}\right)$ with Whitney constant $A$, we conclude that

$$
\left(\left.\partial_{\widehat{v}_{1}}\left(\widehat{P} \odot_{m+1, n+d}^{(x, 0)} \widehat{Q}\right)\right|_{\widehat{v}=0}, \ldots,\left.\partial_{\widehat{v}_{d}}\left(\widehat{P} \odot_{m+1, n+d}^{(x, 0)} \widehat{Q}\right)\right|_{\widehat{v}=0}\right) \in A \sigma(x) .
$$

This together with (3.3) shows that $\widehat{P} \odot{ }_{m+1, n+d}^{(x, 0)} \widehat{Q} \in A \widehat{\sigma}((x, 0))$.

This concludes the proof.

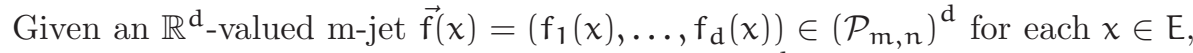
we define, for each point $(x, 0) \in E \times\{0\} \subset \mathbb{R}^{n} \times \mathbb{R}^{\mathrm{d}}$, a real-valued $(\mathrm{m}+1)$-jet $\widehat{f}(x, 0) \in \mathcal{R}_{m+1, n+d}^{(x, 0)}$ by

$$
\widehat{f}(x, 0)(\widehat{x}, \widehat{v})=\sum_{j=1}^{d} \widehat{v}_{j}\left[f_{j}(x)(\widehat{x})\right] .
$$

We will check that the assumption in Theorem 3 is satisfied with $E \times\{0\}, m+1$, $n+d, \widehat{f}(x, 0)$, and $\widehat{\sigma}((x, 0))$ in place of $\bar{E}, m, n, \bar{f}(x)$, and $\bar{\sigma}(x)$, respectively.

Toward this end, we let $S \times\{0\} \subseteq E \times\{0\}$ with $\#(S) \leq k^{\#}$ and show that there exists a map $(x, 0) \longmapsto \widehat{\mathrm{P}}^{(x, 0)}$ from $\mathrm{S} \times\{0\} \rightarrow \mathcal{R}_{\mathrm{m}+1, \mathrm{n}+\mathrm{d}}^{(x, 0)}$ such that

1) $\widehat{P}^{x}-\widehat{f}(x, 0) \in M \widehat{\sigma}((x, 0))$ for all $x \in S$;

2) $\left|\partial^{\gamma} \widehat{\mathrm{P}}^{(x, 0)}(x, 0)\right| \leq M$ for all $x \in S,|\gamma| \leq \mathrm{m}+1$;

3) $\left|\partial^{\gamma}\left(\widehat{P}^{(x, 0)}-\widehat{P}^{(y, 0)}\right)(x, 0)\right| \leq M \omega(|x-y|)|x-y|^{m+1-|\gamma|}$ for $|\gamma| \leq m+1$ and $|x-y| \leq 1, x, y \in S$.

By the assumption of Theorem 1 , there exists $\vec{F}^{S} \in C^{m, \omega}\left(\mathbb{R}^{n}, \mathbb{R}^{d}\right)$ such that (i) $\mathrm{J}_{\chi}^{\mathrm{m}} \overrightarrow{\mathrm{F}}^{\mathrm{S}} \in \mathrm{f}(\mathrm{x})+\mathrm{M \sigma}(\mathrm{x})$ for each $\chi \in S$ and (ii) $\left\|\overrightarrow{\mathrm{F}}^{\mathrm{S}}\right\|_{\mathrm{C}^{\mathrm{m}, \omega}\left(\mathbb{R}^{n}, \mathbb{R}^{\mathrm{d}}\right)} \leq M$.

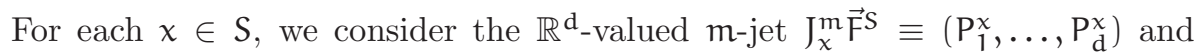
define a real-valued $(m+1)$-jet at $(x, 0)$ by

$$
\widehat{\mathrm{P}}^{(x, 0)}(\widehat{x}, \widehat{v}) \equiv \sum_{j=1}^{\mathrm{d}} \widehat{v}_{j} \mathrm{P}_{j}^{x}(\widehat{x}) .
$$

We will show that $\widehat{P}^{(x, 0)}$ defined above satisfies 1$\left.), 2\right)$ and 3$)$.

By the definitions of $\widehat{f}(x, 0)$ and $\widehat{P}^{(x, 0)}$ (see (3.7) and (3.8)), we have

$$
\left.\left[\widehat{P}^{(x, 0)}-\widehat{f}(x, 0)\right]\right|_{\widehat{v}=0} \equiv 0 .
$$

Furthermore, we have

$$
\begin{aligned}
& \left(\left.\left(\partial_{\widehat{v}_{1}}\left[\widehat{P}^{(x, 0)}-\widehat{f}(x, 0)\right]\right)\right|_{\widehat{v}=0}, \ldots,\left.\left(\partial_{\widehat{v}_{d}}\left[\widehat{P}^{(x, 0)}-\widehat{f}(x, 0)\right]\right)\right|_{\widehat{v}=0}\right) \\
& \quad=\left(P_{1}^{x}-f_{1}^{x}, \ldots, P_{d}^{x}-f_{d}^{x}\right) \in M \sigma(x), \quad \text { since } J_{x}^{m} \vec{F}^{S} \in \vec{f}(x)+M \sigma(x) .
\end{aligned}
$$

This together with (3.9) proves 1). 
For 2) and 3), we write $\partial^{\gamma}=\partial_{\widehat{\chi}}^{\alpha} \partial_{\widehat{v}}^{\beta}$.

From (3.8), we see that

$$
\left.\partial_{\widehat{v}}^{\beta} \widehat{\mathrm{P}}^{(x, 0)}\right|_{\widehat{v}=0} \equiv 0 \quad \text { if }|\beta| \neq 1 .
$$

Thanks to (3.11), we have $\left|\partial_{\widehat{x}}^{\alpha} \partial_{\widehat{v}}^{\beta} \widehat{P}^{(x, 0)}(x, 0)\right|=0$ if $|\beta| \neq 1$; by $(3.8)$, we have

$$
\left|\partial_{\widehat{x}}^{\alpha} \partial_{\widehat{v}}^{\beta} \widehat{P}^{(x, 0)}(x, 0)\right|=\left|\partial_{\widehat{x}}^{\alpha} P_{j}^{x}(x)\right| \leq M
$$

for some $1 \leq j \leq d$ if $|\beta|=1$ and $|\alpha| \leq m$. (Here we used the assumption that $\left\|\vec{F}^{S}\right\|_{C^{m}, \omega\left(\mathbb{R}^{n}, \mathbb{R}^{d}\right)} \leq M$.) To wit, we have $\left|\partial \gamma \widehat{P}^{(x, 0)}(x, 0)\right| \leq M$ for all $x \in S$, $|\gamma| \leq \mathrm{m}+1$. This shows 2).

To prove 3 ), we consider two cases: $|\beta| \neq 1$ and $|\beta|=1$. For $x, y \in S$ and $|\beta| \neq 1$, in view of $(3.11)$, we see that

$$
\left|\partial^{\gamma}\left(\widehat{P}^{(x, 0)}-\widehat{P}^{(y, 0)}\right)(x, 0)\right|=0
$$

which trivially implies 3 ). Now, for $|\beta|=1$ and for all $x, y \in S$ with $|x-y| \leq 1$, we have

$$
\begin{aligned}
& \left|\partial^{\gamma}\left(\widehat{P}^{(x, 0)}-\widehat{P}^{(y, 0)}\right)(x, 0)\right| \\
& \quad=\left|\partial_{\widehat{x}}^{\alpha}\left(P_{j}^{x}-P_{j}^{y}\right)(x)\right| \quad \text { for some } 1 \leq j \leq d, \\
& \quad \leq M \omega(|x-y|)|x-y|^{m-|\alpha|, \quad \text { since }\left\|\vec{F}^{S}\right\|_{C^{m, \omega}\left(\mathbb{R}^{n}, \mathbb{R}^{d}\right)} \leq M,} \\
& \quad \leq M \omega(|x-y|)|x-y|^{m+1-|\gamma|}, \quad \text { since }|\gamma|=1+|\alpha| \text { when }|\beta|=1 .
\end{aligned}
$$

From (3.12) and (3.13), we obtain 3).

We have verified 1), 2) and 3); by Theorem 3, we can conclude that there exists $\mathrm{G} \in \mathrm{C}^{\mathrm{m}+1, \omega}\left(\mathbb{R}^{\mathrm{n}+\mathrm{d}}\right)$ such that

1. $\|\mathrm{G}\|_{\mathrm{C}^{\mathrm{m}+1, \omega}\left(\mathbb{R}^{n+\mathrm{d}}\right)} \leq \mathrm{CM}$;

2. $J_{(x, 0)}^{m+1} G-\widehat{f}(x, 0) \in C M \widehat{\sigma}((x, 0))$ for all $x \in E$.

Here, $\mathrm{C}$ depends only on $A, \boldsymbol{m}, \mathfrak{n}$, and $\mathrm{d}$. By the definition of $\widehat{\sigma}((x, 0))$ and $(3.7)$, we see that $G(\widehat{x}, \widehat{v}): \mathbb{R}^{n+d} \rightarrow \mathbb{R}$ satisfies:

1. $\mathrm{G}(\widehat{x}, 0) \equiv 0$;

2. $\left(J_{x}^{m}\left[\left.\partial_{\widehat{v}_{1}} G\right|_{\widehat{v}_{1}=0}\right], \ldots, J_{x}^{m}\left[\left.\partial_{\widehat{v}_{d}} G\right|_{\widehat{v}=0}\right]\right) \in \vec{f}(x)+M C \sigma(x)$ for all $x \in E$;

3. $\|\mathrm{G}\|_{\mathrm{C}^{\mathrm{m}+1, \omega}\left(\mathbb{R}^{n+\mathrm{d}}\right)} \leq \mathrm{C} M$, for some constant $\mathrm{C}$ depending only on $\mathrm{m}, \mathrm{n}, \mathrm{d}$, and $A$.

In view of the remarks at the beginning of this section, we have proven Theorem 1. 


\section{Proof of the $\mathrm{C}^{\mathrm{m}}$ extension theorem}

In this section, we prove Theorem 2. The relevant terminology is given in the introduction.

Before we embark on the proof of Theorem 2, we recall the following result (Theorem 2 in [12]).

Theorem 4 ( $\mathrm{C}^{\mathrm{m}}$ extension theorem for real-valued functions, C. Fefferman [12]). Let $\overline{\mathrm{E}} \subset \mathbb{R}^{\mathrm{n}}$ be compact. Suppose for each $\mathrm{x} \in \overline{\mathrm{E}}$ we are given an affine subspace $\overline{\mathrm{H}}(x) \subseteq \mathcal{R}_{\mathrm{m}, \mathrm{n}}^{\mathrm{x}}$ having the form $\overline{\mathrm{H}}(\mathrm{x})=\overline{\mathrm{f}}(\mathrm{x})+\overline{\mathrm{I}}(\mathrm{x})$, where $\overline{\mathrm{f}}(\mathrm{x}) \in \mathcal{R}_{\mathrm{m}, \mathrm{n}}^{\mathrm{x}}$ and $\overline{\mathrm{I}}(\mathrm{x})$ is an ideal in $\mathcal{R}_{\mathrm{m}, \mathrm{n}}^{\mathrm{x}}$. Assume that $\{\overline{\mathrm{H}}(\mathrm{x})\}_{\mathrm{x} \in \overline{\mathrm{E}}}$ is its own Glaeser refinement. Then there exists $\overline{\mathrm{F}} \in \mathrm{C}^{\mathrm{m}}\left(\mathbb{R}^{\mathrm{n}}\right)$ with $\mathrm{J}_{\mathrm{x}}^{\mathrm{m}} \overline{\mathrm{F}} \in \overline{\mathrm{H}}(\mathrm{x})$ for all $\mathrm{x} \in \mathrm{E}$.

Remark 2. In [12], the first author showed that the large constant $k^{\#}$ appearing implicitly in Theorem 4 can be bounded by a constant depending only on $m$ and $n$. Later, in [1], Bierstone-Milman gave a sharper upper bound on $k^{\#}$ in the case $\overline{\mathrm{I}}(x) \equiv\{0\}$. In that case, they showed that $\mathrm{k}^{\#}=2^{\operatorname{dim}\left(\mathcal{P}_{m, n}\right)}$ is sufficient. Compare with Remark 1.

Proof of Theorem 2. We recall that each fiber of the given bundle $\overrightarrow{\mathcal{H}}=(\overrightarrow{\mathrm{H}}(x))_{x \in \mathrm{E}}$ takes the form

$$
\overrightarrow{\mathrm{H}}(x)=\overrightarrow{\mathrm{P}}^{x}+\overrightarrow{\mathrm{I}}(x),
$$

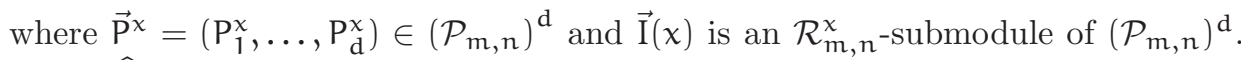
Define $\widehat{f}: E \times\{0\} \subset \mathbb{R}^{n} \times \mathbb{R}^{\mathrm{d}} \rightarrow \mathcal{P}_{\mathrm{m}+1, \mathrm{n}+\mathrm{d}}$ by

$$
\widehat{f}((x, 0))=\sum_{j=1}^{d} \widehat{v}_{j} P_{j}^{x}(\widehat{x}) \in \mathcal{R}_{m+1, n+d}^{(x, 0)} .
$$

For each $(x, 0) \in E \times\{0\}$, consider the set

$$
\begin{aligned}
\widehat{I}((x, 0))=\left\{P \in \mathcal{R}_{m+1, n+d}^{(x, 0)}:\right. & P(\widehat{x}, 0) \equiv 0, \\
& \left.\left(\left.\left[\partial_{\widehat{v}_{1}} P(\widehat{x}, \widehat{v})\right]\right|_{\widehat{v}=0}, \ldots,\left.\left[\partial_{\widehat{v}_{d}} P(\widehat{x}, \widehat{v})\right]\right|_{\widehat{v}=0}\right) \in \vec{I}(x)\right\} .
\end{aligned}
$$

Lemma 4.1. For each $(x, 0) \in \mathrm{E} \times\{0\}, \widehat{\mathrm{I}}((x, 0))$ is an ideal in $\mathcal{R}_{\mathrm{m}+1, \mathrm{n}+\mathrm{d}}^{(\mathrm{x}, 0)}$.

Proof of Lemma 4.1. Let $\mathrm{P} \in \widehat{\mathrm{I}}((x, 0))$ and $\mathrm{Q} \in \mathcal{R}_{\mathrm{m}+1, \mathrm{n}+\mathrm{d}}^{(\mathrm{x}, 0)}$. We must show that

$$
\left[P \odot{ }_{m+1, n+d}^{(x, 0)} Q\right] \in \widehat{I}((x, 0)),
$$

where $\odot_{\mathrm{m}+1, \mathrm{n}+\mathrm{d}}^{(x, 0)}$ denotes the multiplication in $\mathcal{R}_{\mathrm{m}+1, \mathrm{n}+\mathrm{d}}^{(x, 0)}$.

Since $\mathrm{P}(\widehat{x}, 0) \equiv 0$, we have

$$
\left.\left.\left.\left[\mathrm{P} \odot \odot_{\mathrm{m}+1, n+\mathrm{d}}^{(x, 0)} \mathrm{Q}\right]\right|_{\widehat{v}=0} \equiv \mathrm{P}\right|_{\widehat{v}=0} \odot_{\mathrm{m}+1, n}^{x} \mathrm{Q}\right|_{\widehat{v}=0} \equiv 0 .
$$


Now, let $\pi_{\mathrm{m}}: \mathcal{R}_{\mathrm{m}+1, \mathrm{n}+\mathrm{d}}^{(\mathrm{x}, 0)} \rightarrow \mathcal{R}_{\mathrm{m}, \mathrm{n}+\mathrm{d}}^{(\mathrm{x}, 0)}$ be the natural projection. We have

$$
\begin{aligned}
& \left(\left.\partial_{\widehat{v}_{1}}\left(\mathrm{P} \odot \odot_{\mathrm{m}+1, n+d}^{(x, 0)} \mathrm{Q}\right)\right|_{\widehat{v}=0}, \ldots,\left.\partial_{\widehat{v}_{\mathrm{d}}}\left(\mathrm{P} \odot_{\mathrm{m}+1, n+d}^{(x, 0)} \mathrm{Q}\right)\right|_{\widehat{v}=0}\right) \\
& =\left(\left.\left.\partial_{\widehat{v}_{1}} \mathrm{P}\right|_{\widehat{v}=0} \odot_{m, n}^{x} \pi_{m} \mathrm{Q}\right|_{\widehat{v}=0}+\left.\left.\pi_{m} \mathrm{P}\right|_{\widehat{v}=0} \odot_{m, n}^{x} \partial_{\widehat{v}} \mathrm{Q}\right|_{\widehat{v}=0}, \ldots,\right. \\
& \left.\left.\left.\partial_{\widehat{v}_{\mathrm{d}}} \mathrm{P}\right|_{\widehat{v}=0} \odot_{\mathrm{m}, \mathrm{n}}^{\mathrm{x}} \pi_{\mathrm{m}} \mathrm{Q}\right|_{\widehat{v}=0}+\left.\left.\pi_{\mathrm{m}} \mathrm{P}\right|_{\widehat{v}=0} \odot_{\mathrm{m}, \mathrm{n}}^{\mathrm{x}} \partial_{\widehat{v}_{\mathrm{d}}} \mathrm{Q}\right|_{\widehat{v}=0}\right) \\
& =\left(\left.\left.\partial_{\widehat{v}} \mathrm{P}\right|_{\widehat{v}=0} \odot_{\mathrm{m}, \mathrm{n}}^{\mathrm{x}} \pi_{\mathrm{m}} \mathrm{Q}\right|_{\widehat{v}=0}, \ldots,\left.\left.\partial_{\widehat{v}} \mathrm{P}\right|_{\widehat{v}=0} \odot_{\mathrm{m}, n}^{x} \pi_{\mathrm{m}} \mathrm{Q}\right|_{\widehat{v}=0}\right) \in \overrightarrow{\mathrm{I}}(x),
\end{aligned}
$$

where we used the facts that $\mathrm{P} \in \widehat{\mathrm{I}}((x, 0))$ (so that $\left.\pi_{\mathrm{m}} P\right|_{\widehat{v}=0} \equiv 0$ ) and that $\overrightarrow{\mathrm{I}}(x)$ is an $\mathcal{R}_{\mathrm{m}, \mathrm{n}}^{\mathrm{x}}$-submodule of $\left(\mathcal{P}_{\mathrm{m}, \mathrm{n}}^{\mathrm{x}}\right)^{\mathrm{d}}$.

From (4.3) and (4.4), we conclude the proof of (4.2).

Remark 3. We recall that $\{\vec{H}(x)\}_{x \in E}$ is assumed to be its own Glaeser refinement. We will show that the bundle

$$
\{\widehat{\mathrm{H}}((x, 0)):=\widehat{f}((x, 0))+\widehat{\mathrm{I}}((x, 0))\}_{(x, 0) \in \mathrm{E} \times\{0\}} \cdot
$$

is also its own Glaeser refinement.

With $n+d, m+1, E \times\{0\}, \widehat{f}, \widehat{I}((x, 0))$, and $\{\widehat{H}((x, 0))\}_{(x, 0) \in E \times\{0\}}$ in place of $n, m$, $\overline{\mathrm{E}}, \overline{\mathrm{f}}, \overline{\mathrm{I}}(x)$, and $\{\overline{\mathrm{H}}(x)\}_{x \in \overline{\mathrm{E}}}$ in Theorem 4 , we see that there exists $\mathrm{G} \in \mathrm{C}^{\mathrm{m}+1}\left(\mathbb{R}^{\mathrm{n}+\mathrm{d}}\right)$ with $\mathrm{J}_{(x, 0)}^{\mathrm{m}+1}(\mathrm{G}) \in \widehat{\mathrm{H}}((x, 0))$ for all $(x, 0) \in E \times\{0\}$.

Setting

$$
\vec{F}(\widehat{x})=\left(\left.\left[\partial_{\widehat{v}_{1}} G(\widehat{x}, \widehat{v})\right]\right|_{\widehat{v}=0}, \ldots,\left.\left[\partial_{\widehat{v}_{d}} G(\widehat{x}, \widehat{v})\right]\right|_{\widehat{v}=0}\right)
$$

in view of the definition of $\widehat{H}((x, 0))$, we have $J_{x}^{m} \vec{F} \in \vec{H}(x)$ for all $x \in E$, thus proving Theorem 2.

Therefore, the crux of the matter is to verify (4.5). To this end, let $x_{0} \in E$ and $\widehat{\mathrm{P}}_{0} \in \widehat{\mathrm{H}}\left(\left(x_{0}, 0\right)\right)$. We will prove that $\widehat{\mathrm{P}}_{0} \in \widehat{\mathrm{H}}^{\prime}\left(\left(x_{0}, 0\right)\right)$.

Since $\widehat{P}_{0} \in \widehat{H}\left(\left(x_{0}, 0\right)\right)$, we can write

$$
\widehat{P}_{0}(\widehat{x}, \widehat{v})=\widehat{f}\left(\left(x_{0}, 0\right)\right)(\widehat{x}, \widehat{v})+\sum_{1 \leq|\xi| \leq m+1} \frac{1}{\xi !} \widehat{v}^{\xi} P_{\xi}(\widehat{x}),
$$

where $P_{\xi} \in \mathcal{P}_{m, n}$ with

$$
\begin{aligned}
\overrightarrow{\mathrm{P}} \equiv\left(\left.\left(\partial_{\widehat{v}_{1}}\left[\sum_{1 \leq|\xi| \leq m+1} \frac{1}{\xi !} \widehat{v}^{\xi} P_{\xi}(\widehat{x})\right]\right)\right|_{\widehat{v}=0}, \ldots\right. \\
\left.\ldots,\left.\left(\partial_{\widehat{v}_{d}}\left[\sum_{1 \leq|\xi| \leq m+1} \frac{1}{\xi !} \widehat{v}^{\xi} P_{\xi}(\widehat{x})\right]\right)\right|_{\widehat{v}=0}\right) \in \overrightarrow{\mathrm{I}}(x) .
\end{aligned}
$$

We write

$$
\overrightarrow{\mathrm{P}}_{0} \equiv\left(\mathrm{P}_{0,1}, \ldots, \mathrm{P}_{0, \mathrm{~d}}\right) \equiv \overrightarrow{\mathrm{P}}^{x_{0}}+\overrightarrow{\mathrm{P}}
$$


Thanks to (4.7), we have

$$
\overrightarrow{\mathrm{P}}_{0} \in \overrightarrow{\mathrm{H}}\left(x_{0}\right) \text {. }
$$

From (4.6), we have

$$
\widehat{P}_{0}(\widehat{x}, \widehat{v})=\sum_{1 \leq k \leq d} \widehat{v}_{k} P_{0, k}(\widehat{x})+\sum_{2 \leq|\xi| \leq m+1} \frac{1}{\xi !} \widehat{v}^{\xi} P_{\xi}(\widehat{x}) .
$$

Fix $\varepsilon>0$. Since $\left\{\vec{H}(x)=\overrightarrow{\mathrm{P}}^{x}+\overrightarrow{\mathrm{I}}(x)\right\}_{x \in E}$ is its own Glaeser refinement, we know that there exists $\delta>0$ such that for all $x_{1}, \ldots, x_{k \#} \in E \cap B\left(x_{0}, \delta\right)$, there exist

$$
\vec{P}_{1}=\left(P_{1,1}, \ldots, P_{1, d}\right) \in \vec{H}\left(x_{1}\right), \ldots, \vec{P}_{k \#}=\left(P_{k \#, 1}, \ldots, P_{k \#, d}\right) \in \vec{H}\left(x_{k \#}\right)
$$

with

$$
\left|\partial^{\alpha}\left(P_{i, k}-P_{j, k}\right)\left(x_{i}\right)\right| \leq \varepsilon\left|x_{i}-x_{j}\right|^{m-|\alpha|}
$$

for $|\alpha| \leq m, 0 \leq i, j \leq k^{\#}$ and $1 \leq k \leq d$.

Now, for any $\left(x_{1}, 0\right), \ldots,\left(x_{k \#}, 0\right) \in(E \times\{0\}) \cap B\left(\left(x_{0}, 0\right), \delta\right)$, we claim the following:

$$
\text { a) } \begin{aligned}
\widehat{P}_{1}(\widehat{x}, \widehat{v}) \equiv & \sum_{j=1}^{d} \widehat{v}_{j} P_{1, j}(\widehat{x})+\sum_{2 \leq|\xi| \leq m+1} \frac{1}{\xi !} \widehat{v}^{\xi} P_{\xi}(\widehat{x}) \in \widehat{H}\left(\left(x_{1}, 0\right)\right), \\
& \vdots \\
\widehat{P}_{k \#}(\widehat{x}, \widehat{v}) \equiv & \sum_{j=1}^{d} \widehat{v}_{j} P_{k \#, j}(\widehat{x})+\sum_{2 \leq|\xi| \leq m+1} \frac{1}{\xi !} \widehat{v}^{\xi} P_{\xi}(\widehat{x}) \in \widehat{H}\left(\left(x_{k \#}, 0\right)\right),
\end{aligned}
$$

where $\overrightarrow{\mathrm{P}}_{1}, \ldots, \overrightarrow{\mathrm{P}}_{\mathrm{k} \#}$ are as chosen in (4.10); $\mathrm{P}_{\xi}$ are as in (4.6).

b) For all $|\gamma| \leq m+1$, we have

$$
\left|\partial^{\gamma}\left(\widehat{P}_{i}-\widehat{P}_{j}\right)\left(\left(x_{i}, 0\right)\right)\right| \leq \varepsilon\left|x_{i}-x_{j}\right|^{m+1-|\gamma|}, \quad \text { for } 0 \leq i, j \leq k^{\#} .
$$

To see a), fix an integer $i \in\left\{1, \ldots, k^{\#}\right\}$ and consider

$$
\begin{aligned}
\widehat{P}_{i}(\widehat{x}, \widehat{v})-\widehat{f} & \left(\left(x_{i}, 0\right)\right)(\widehat{x}, \widehat{v}) \\
& =\left(\sum_{j=1}^{d} \widehat{v}_{j} P_{i, j}(\widehat{x})+\sum_{2 \leq|\xi| \leq m+1} \frac{1}{\xi !} \widehat{v}^{\xi} P_{\xi}(\widehat{x})\right)-\sum_{j=1}^{d} \widehat{v}_{j} P_{j}^{x_{i}}(\widehat{x}) \\
& =\sum_{j=1}^{d} \widehat{v}_{j}\left(P_{i, j}-P_{j}^{x_{i}}\right)(\widehat{x})+\sum_{2 \leq|\xi| \leq m+1} \frac{1}{\xi !} \widehat{v}^{\xi} P_{\xi}(\widehat{x}) .
\end{aligned}
$$

Notice that

$$
\left.\left[\partial_{\widehat{v}}^{\beta}\left(\sum_{2 \leq|\xi| \leq m+1} \frac{1}{\xi !} \widehat{v}^{\xi} P_{\xi}(\widehat{x})\right)\right]\right|_{\widehat{v}=0} \equiv 0 \quad \text { for }|\beta| \leq 1 .
$$


From (4.13) and (4.14), it follows that

$$
\left[\widehat{P}_{i}(\widehat{x}, 0)-\widehat{f}\left(\left(x_{i}, 0\right)\right)\right](\widehat{x}, 0) \equiv 0
$$

Next, we have

$$
\begin{gathered}
\left(\left.\left[\partial_{\widehat{v}_{1}}\left(\widehat{P}_{i}-\widehat{f}\left(\left(x_{i}, 0\right)\right)\right)(\widehat{x}, \widehat{v})\right]\right|_{\widehat{v}=0}, \ldots,\left.\left[\partial_{\widehat{v}_{d}}\left(\widehat{P}_{i}-\widehat{f}\left(\left(x_{i}, 0\right)\right)\right)(\widehat{x}, \widehat{v})\right]\right|_{\widehat{v}=0}\right) \\
=\left(\left(P_{i, 1}-P_{1}^{x_{i}}\right)(\widehat{x}), \ldots,\left(P_{i, d}-P_{d}^{x_{i}}\right)(\widehat{x})\right) \in \vec{I}\left(x_{i}\right),
\end{gathered}
$$

where the first equality follows from (4.13) and (4.14); and the last relation follows from (4.10). Together with (4.15), this completes the proof of a).

For b), we write $\partial^{\gamma}=\partial_{\widehat{\chi}}^{\alpha} \partial_{\widehat{v}}^{\beta}$ and observe that for $0 \leq i \leq k^{\#}$,

$$
\left.\left[\partial_{\widehat{v}} \widehat{P}_{i}\right]\right|_{\widehat{v}=0} \equiv\left\{\begin{array}{cl}
0 & \text { if }|\beta|=0 \\
P_{i, j} & \text { for some } 1 \leq j \leq d, \text { if }|\beta|=1 . \\
P_{\beta} & |\beta| \geq 2
\end{array} .\right.
$$

In view of (4.16), we see that (4.12) holds trivially for $|\beta| \neq 1$. Therefore, it suffices to show (4.12) for $|\beta|=1$. Without loss of generality, we may assume $\partial_{\widehat{v}}^{\beta}=\partial_{\widehat{v}_{k}}$ for some $k \in\{1, \ldots, d\}$. We have

$$
\begin{aligned}
\left|\partial^{\gamma}\left(\widehat{P}_{i}-\widehat{P}_{j}\right)\left(\left(x_{i}, 0\right)\right)\right| & =\left|\partial_{\widehat{x}}^{\alpha} \partial_{\widehat{v}_{k}}\left(\widehat{P}_{i}-\widehat{P}_{j}\right)\left(\left(x_{i}, 0\right)\right)\right| \\
& =\left|\partial_{\widehat{x}}^{\alpha}\left(P_{i, k}-P_{j, k}\right)\left(x_{j}\right)\right| \\
& \leq \varepsilon\left|x_{i}-x_{j}\right|^{m-|\alpha|}, \text { thanks to (4.11), } \\
& =\varepsilon\left|x_{i}-x_{j}\right|^{m+1-|\gamma|},
\end{aligned}
$$

establishing (4.12).

Properties a) and $b)$ show that $\widehat{P}_{0} \in \widehat{H}^{\prime}\left(\left(x_{0}, 0\right)\right)$. Since $x_{0} \in E$ and $\widehat{P}_{0} \in$ $\widehat{H}\left(\left(x_{0}, 0\right)\right)$ are arbitrary, this completes the proof of $(4.5)$. Theorem 2 now follows from Remark 3.

\section{Solutions to Problem 1 for $X=C^{m}\left(\mathbb{R}^{\mathfrak{n}}\right)$ and $X=C^{m, \omega}\left(\mathbb{R}^{\mathfrak{n}}\right)$}

Armed with Theorems 1 and 2, we are now in a position to answer Problem 1 for $X=C^{m}\left(\mathbb{R}^{n}\right)$ and $X=C^{m, \omega}\left(\mathbb{R}^{n}\right)$.

We write $Q^{\circ}=[-1 / 2,1 / 2]^{n}$ to denote the unit cube in $\mathbb{R}^{n}$.

For $\mathrm{X}=\mathrm{C}^{\mathrm{m}, \omega}\left(\mathrm{Q}^{\mathrm{o}}\right)$, to apply Theorem 1 , we will take $\mathrm{E}=\mathrm{Q}^{\mathrm{o}}$ and

$$
\sigma(x)=\left\{P \in\left(\mathcal{P}_{m, n}\right)^{d}: \sum_{i=1}^{d} P_{i}(x) f_{i}(x)=0\right\} .
$$

It is easy to see that $\sigma(x)$ is Whitney $\omega$-convex with Whitney constant 1. By Theorem 1 and the standard Whitney extension Theorem for $C^{m, \omega}\left(\mathbb{R}^{n}\right.$ ) (see Theorem 3), we easily see that the solvability of Problem 1 for $X=C^{m, \omega}\left(Q^{o}\right)$ is equivalent to the solvability of the following elementary linear algebra problem: 
Does there exist $\mathrm{M}<\infty$ such that the following holds: Given any $\mathrm{k}^{\#}$ distinct points $\mathrm{x}_{1}, \ldots, \mathrm{x}_{\mathrm{k} \#} \in \mathrm{Q}^{\mathrm{o}}$, there exist $\mathrm{P}_{1}^{\mathrm{j}}, \ldots, \mathrm{P}_{\mathrm{k} \#}^{\mathrm{j}} \in \mathcal{P}_{\mathrm{m}, \mathrm{n}}$ for $1 \leq \mathrm{j} \leq \mathrm{d}$ such that

$$
\left\{\begin{array}{l}
\sum_{j=1}^{\mathrm{d}} f_{j}\left(x_{k}\right) P_{k}^{j}\left(x_{k}\right)=\phi\left(x_{k}\right) \text { for } k=1, \ldots, k^{\#} \\
\sum_{k=1}^{k^{\#}} \sum_{j=1}^{d} \sum_{|\alpha| \leq m}\left|\partial^{\alpha} P_{k}^{j}\left(x_{k}\right)\right|^{2} \\
\quad+\sum_{1 \leq k<k^{\prime} \leq k \#} \sum_{j=1}^{d} \sum_{|\alpha| \leq m}\left|\frac{\partial^{\alpha}\left(P_{k}^{j}-P_{k^{\prime}}^{j}\right)\left(x_{k}\right)}{\omega\left(\left|x_{k}-x_{k^{\prime}}\right|\right)\left|x_{k}-x_{k^{\prime}}\right|^{m-|\alpha|}}\right|^{2} \leq M^{2}
\end{array}\right.
$$

Next, we describe the solution to Problem 1 for $X=C^{m}\left(Q^{\circ}\right)$.

It is easy to see that the m-jet of any $\mathrm{C}^{\mathrm{m}}\left(\mathrm{Q}^{\mathrm{o}}\right)$ solution of Problem 1 at $x \in \mathrm{Q}^{\circ}$ belongs to

$$
\vec{H}(x)=\left\{\vec{P}=\left(P_{1}, \ldots, P_{d}\right) \in\left(\mathcal{P}_{m, n}\right)^{d}: \sum_{i=1}^{d} P_{i}(x) f_{i}(x)=\phi(x)\right\} .
$$

We consider the bundle $\overrightarrow{\mathcal{H}}=(\vec{H}(x))_{x \in Q^{\circ}}$. Solving Problem 1 amounts to deciding whether this bundle admits a section.

By Taylor's theorem, we know that the sections of a bundle $\overrightarrow{\mathcal{H}}$ coincide with the sections of its Glaeser refinement.

Now we describe an effective method for computing the Glaeser refinements.

Given points $x_{0}, x_{1}, \ldots, x_{k} \in \mathbb{R}^{n}$, and given polynomials $\overrightarrow{\mathrm{P}}_{0}=\left(\overrightarrow{\mathrm{P}}_{0,1}, \ldots, \overrightarrow{\mathrm{P}}_{0, \mathrm{~d}}\right)$, $\vec{P}_{1}=\left(\vec{P}_{1,1}, \ldots, \vec{P}_{1, d}\right), \ldots, \vec{P}_{k}=\left(\vec{P}_{k, 1}, \ldots, \vec{P}_{k, d}\right) \in\left(\mathcal{P}_{m, n}\right)^{d}$, we define

$$
\mathcal{Q}\left(\vec{P}_{0}, \vec{P}_{1}, \ldots, \vec{P}_{k} ; x_{0}, x_{1}, \ldots, x_{k}\right) \equiv \sum_{\substack{i^{\prime}, i=0 \\ x_{i} \neq x_{i^{\prime}}}}^{k} \sum_{j=1}^{d} \sum_{|\alpha| \leq m}\left|\frac{\partial^{\alpha}\left(P_{i, j}-P_{i^{\prime}, j}\right)\left(x_{i}\right)}{\left|x_{i}-x_{i^{\prime}}\right| m-|\alpha|}\right|^{2} .
$$

Fix $x_{0} \in Q^{o}$ and $\vec{P}_{0} \in \vec{H}\left(x_{0}\right)$. For fixed $x_{1}, \ldots, x_{k}^{\#} \in Q^{o}$, we compute the minimum for the following quadratic form over a finite-dimensional affine space:

$$
\begin{aligned}
& \operatorname{MIN}\left(x_{0}, \overrightarrow{\mathrm{P}}_{0} ; x_{1}, \ldots, x_{\mathrm{k}}^{\#}\right) \\
& \quad \equiv \min \left\{\mathcal{Q}\left(\overrightarrow{\mathrm{P}}_{0}, \overrightarrow{\mathrm{P}}_{1}, \ldots, \overrightarrow{\mathrm{P}}_{\mathrm{k} \#} ; x_{0}, x_{1}, \ldots, x_{\mathrm{k} \#}\right): \overrightarrow{\mathrm{P}}_{1} \in \overrightarrow{\mathrm{H}}\left(x_{1}\right), \ldots, \overrightarrow{\mathrm{P}}_{\mathrm{k} \#} \in \overrightarrow{\mathrm{H}}\left(x_{\mathrm{k} \#}\right)\right\} .
\end{aligned}
$$

Determining $\operatorname{MIN}\left(x_{0}, \vec{P}_{0} ; x_{1}, \ldots, x_{k}^{\#}\right)$ is just a routine linear algebra problem. From the definition of Glaeser refinement, it is easy to see that given $x_{0} \in Q^{\circ}$ and $\overrightarrow{\mathrm{P}}_{0} \in \overrightarrow{\mathrm{H}}\left(x_{0}\right)$, we have $\overrightarrow{\mathrm{P}}_{0} \in \overrightarrow{\mathrm{H}}^{\prime}\left(x_{0}\right)$ if and only if

$$
\limsup _{\delta \downarrow 0, x_{1}, \ldots, x_{k}^{\#} \in B\left(x_{0}, \delta\right)} \operatorname{MIN}\left(x_{0}, \vec{P}_{0} ; x_{1}, \ldots, x_{k}^{\#}\right)=0 .
$$

To wit, computing the Glaeser refinement of a bundle $\overrightarrow{\mathcal{H}}$ involves doing elementary linear algebra and calculating the limsup.

The following lemma states that the Glaeser refinements stabilize after a finite number of iterations. 
Lemma 5.1 (Stabilization Lemma adapted from [12] (which in turn is adapted from [14] and [2])). Let $\mathrm{E} \subset \mathbb{R}^{\mathrm{n}}$ be compact. Suppose we are given a bundle $\overrightarrow{\mathcal{H}}_{0}=\{\overrightarrow{\mathrm{H}}(\mathrm{x})\}_{x \in \mathrm{E}}$. For $\mathrm{l} \geq 0$, let $\overrightarrow{\mathcal{H}}_{\mathrm{l+1}}=\left\{\overrightarrow{\mathrm{H}}_{\mathrm{l}+1}(\mathrm{x})\right\}_{\mathrm{x} \in \mathrm{E}}$ be the Glaeser refinement of $\overrightarrow{\mathcal{H}}_{\mathrm{l}}=\left\{\overrightarrow{\mathrm{H}}_{\mathrm{l}}(\mathrm{x})\right\}_{\mathrm{x} \in \mathrm{E}}$. For each $\mathrm{x} \in \mathrm{E}$, if $\operatorname{dim} \overrightarrow{\mathrm{H}}_{2 \mathrm{k}+1}(\mathrm{x}) \geq \operatorname{dim}\left[\left(\mathcal{P}_{\mathrm{m}, \mathrm{n}}\right)^{\mathrm{d}}\right]-\mathrm{k}$, then $\overrightarrow{\mathrm{H}}_{\mathrm{l}}(\mathrm{x})=\overrightarrow{\mathrm{H}}_{2 \mathrm{k}+1}(\mathrm{x})$ for all $\mathrm{l} \geq 2 \mathrm{k}+1$.

Proof of Lemma 5.1. Fix $x \in E$. We proceed by induction on $k$. For $k=0$, the lemma asserts that

$$
\text { if } \overrightarrow{\mathrm{H}}_{1}(x)=\left(\mathcal{P}_{\mathrm{m}, \mathrm{n}}\right)^{\mathrm{d}} \text {, then } \overrightarrow{\mathrm{H}}_{\mathrm{l}}(x)=\overrightarrow{\mathrm{H}}_{1}(x) \text { for all } \mathrm{l} \geq 1 \text {. }
$$

From the definition of $\overrightarrow{\mathcal{H}}_{l}$, one sees that

$$
\operatorname{dim} \overrightarrow{\mathrm{H}}_{l+1}(x) \leq \lim _{y \rightarrow x} \inf \operatorname{dim} \overrightarrow{\mathrm{H}}_{l}(\mathrm{y}) .
$$

Hence, if $\overrightarrow{\mathrm{H}}_{1}(x)=\left(\mathcal{P}_{\mathrm{m}, \mathrm{n}}\right)^{\mathrm{d}}$, then $\overrightarrow{\mathrm{H}}_{\mathrm{o}}(\mathrm{y})=\left(\mathcal{P}_{\mathrm{m}, \mathrm{n}}\right)^{\mathrm{d}}$ for all $\mathrm{y}$ in a neighborhood of $x$. Consequently, $\vec{H}_{l}(y)=\left(\mathcal{P}_{m, n}\right)^{\mathrm{d}}$, for all $\mathrm{l} \geq 1$, proving (5.1).

For the induction step, fix some $k \geq 0$ and assume Lemma 5.1 holds for that $k$. We must show that

$$
\text { if } \begin{aligned}
\operatorname{dim} \overrightarrow{\mathrm{H}}_{2 k+3}(x) \geq \operatorname{dim}\left[\left(\mathcal{P}_{\mathrm{m}, \mathrm{n}}\right)^{\mathrm{d}}\right]-(\mathrm{k}+1), \\
\text { then } \overrightarrow{\mathrm{H}}_{l}(x)=\overrightarrow{\mathrm{H}}_{2 k+3}(x) \text { for all } l \geq 2 k+3 .
\end{aligned}
$$

By the inductive hypothesis, we know that if $\operatorname{dim} \vec{H}_{2 k+1}(x) \geq \operatorname{dim}\left[\left(\mathcal{P}_{m, n}\right)^{d}\right]-k$, then $\vec{H}_{l}(x)=\vec{H}_{2 k+1}(x)$ for all $l \geq 2 k+1$; consequently, (5.3) holds. Thus, to prove (5.3), we may assume that

$$
\operatorname{dim} \overrightarrow{\mathrm{H}}_{2 \mathrm{k}+1}(x) \leq \operatorname{dim}\left[\left(\mathcal{P}_{\mathrm{m}, \mathrm{n}}\right)^{\mathrm{d}}\right]-(\mathrm{k}+1) .
$$

Thus,

(5.4) $\operatorname{dim} \overrightarrow{\mathrm{H}}_{2 k+1}(x)=\operatorname{dim} \overrightarrow{\mathrm{H}}_{2 \mathrm{k}+2}(x)=\operatorname{dim} \overrightarrow{\mathrm{H}}_{2 \mathrm{k}+3}(x)=\operatorname{dim}\left[\left(\mathcal{P}_{\mathrm{m}, \mathrm{n}}\right)^{\mathrm{d}}\right]-(\mathrm{k}+1)$.

Note that $\operatorname{dim} \overrightarrow{\mathrm{H}}_{2 \mathrm{k}+1}(\mathrm{y}) \geq \operatorname{dim}\left(\mathcal{P}_{\mathrm{m}, \mathrm{n}}\right)^{\mathrm{d}}-(\mathrm{k}+1)$ for all $\mathrm{y}$ sufficiently close to $x$ since otherwise $(5.2)$ (with $l=2 k+1$ ) would contradict (5.4).

Next, we will show the following:

$$
\overrightarrow{\mathrm{H}}_{2 \mathrm{k}+2}(\mathrm{y})=\overrightarrow{\mathrm{H}}_{2 \mathrm{k}+1}(\mathrm{y}) \text { for all } y \text { sufficiently close to } x \text {. }
$$

Suppose toward a contradiction that (5.5) fails; that is,

(5.6) there exists $y$ arbitrarily near $x$ such that $\operatorname{dim} \vec{H}_{2 k+2}(y) \leq \operatorname{dim} \vec{H}_{2 k+1}(y)-1$.

Then, since we are assuming Lemma 5.1 for $k$, we must have

$$
\operatorname{dim} \vec{H}_{2 k+1}(y) \leq \operatorname{dim}\left[\left(\mathcal{P}_{m, n}\right)^{d}\right]-(k+1)
$$

for all $y$ as in (5.6). 
This together with (5.6) shows

(5.8) $\operatorname{dim} \overrightarrow{\mathrm{H}}_{2 \mathrm{k}+2}(\mathrm{y}) \leq \operatorname{dim}\left[\left(\mathcal{P}_{\mathrm{m}, \mathrm{n}}\right)^{\mathrm{d}}\right]-\mathrm{k}-2$ for all $\mathrm{y}$ arbitrarily close to $x$. From (5.2) and (5.8), we get

$$
\operatorname{dim} \overrightarrow{\mathrm{H}}_{2 \mathrm{k}+3}(x) \leq \operatorname{dim}\left[\left(\mathcal{P}_{\mathrm{m}, \mathrm{n}}\right)^{\mathrm{d}}\right]-\mathrm{k}-2,
$$

contradicting (5.4). Therefore, (5.5) cannot fail.

By (5.5), we see easily that $\vec{H}_{l}(y)=\vec{H}_{2 k+1}(y)$ for all $l \geq 2 k+1$ and all $y \in E$ sufficiently close to $x$. In particular, we have $\vec{H}_{l}(x)=\vec{H}_{2 k+3}(x)$ for all $l \geq 2 k+3$. This completes the induction step, and proves Lemma 5.1.

According to this lemma, we know that $\overrightarrow{\mathcal{H}}_{l}=\overrightarrow{\mathcal{H}}_{2 \operatorname{dim}\left[\left(\mathcal{P}_{m, n}\right) \mathrm{d}\right]+1}$ for

$$
l \geq 2 \operatorname{dim}\left[\left(\mathcal{P}_{\mathrm{m}, \mathrm{n}}\right)^{\mathrm{d}}\right]+1
$$

Moreover, following the argument for Lemma 2.1 in [12], we see that if $\vec{H}(x)=$

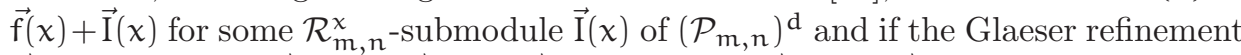
$\vec{H}^{\prime}(x) \neq \emptyset$, then $\vec{H}^{\prime}(x)=\vec{f}_{1}(x)+\vec{I}_{1}(x)$ for some $\vec{f}_{1}(x) \in \vec{H}^{\prime}(x)$ and some $\mathcal{R}_{m, n^{-}}^{x}$ submodule $\overrightarrow{\mathrm{I}}_{\mathrm{l}}(\mathrm{x})$ of $\left(\mathcal{P}_{\mathrm{m}, \mathrm{n}}\right)^{\mathrm{d}}$. Consequently, by Theorem 2 , we easily see that Problem 1 is solvable for $X=C^{m}\left(\mathbb{R}^{n}\right)$ if and only if the bundle $\overrightarrow{\mathcal{H}}_{2 \operatorname{dim}\left[\left(\mathcal{P}_{m, n}\right)^{\mathrm{d}}\right]+1}$ contains no empty fiber.

\section{References}

[1] Bierstone, E. and Milman, P.D.: $C^{m}$-norms on finite sets and $C^{m}$ extension criteria. Duke Math. J. 137 (2007), no. 1, 1-18.

[2] Bierstone, E., Milman, P. D. And PawŁucki, W.: Differentiable functions defined in closed sets. A problem of Whitney. Invent. Math. 151 (2003), no. 2, 329-352.

[3] Brenner, H.: Continuous solutions to algebraic forcing equations. Preprint available at arXiv: math/0608611v2, 2006.

[4] Brudnyı̆, Yu. A. and Shvartsman, P.: A linear extension operator for a space of smooth functions defined on a closed subset in $\mathbb{R}^{n}$. Dokl. Akad. Nauk SSSR 280 (1985), no. 2, 268-272.

[5] Brudny̆̌, Yu. A. And Shvartsman, P.: Generalizations of Whitney's extension theorem. Internat. Math. Res. Notices 1994, no. 3, 129 ff., approx. 11 pp. (electronic).

[6] Brudny, Yu. A. and Shvartsman, P.: The Whitney problem of existence of a linear extension operator. J. Geom. Anal. 7 (1997), no. 4, 515-574.

[7] Brudny̆, Yu. A. And Shvartsman, P.: Whitney's extension problem for multivariate $C^{1, \omega}$-functions. Trans. Amer. Math. Soc. 353 (2001), no. 6, 2487-2512 (electronic).

[8] Epstein, N. and Hochster, M.: Continuous closure, axes closure, and natural closure. Preprint available at arXiv: 1106.3462v1, June 2011. 
[9] Fefferman, C.: A generalized sharp Whitney theorem for jets. Rev. Mat. Iberoamericana 21 (2005), no. 2, 577-688.

[10] Fefferman, C.: Interpolation and extrapolation of smooth functions by linear operators. Rev. Mat. Iberoamericana 21 (2005), no. 1, 313-348.

[11] Fefferman, C.: A sharp form of Whitney's extension theorem. Ann. of Math. (2) 161 (2005), no. 1, 509-577.

[12] Fefferman, C.: Whitney's extension problem for $\mathrm{C}^{\mathrm{m}}$. Ann. of Math. (2) 164 (2006), no. 1, 313-359.

[13] Fefferman, C. and Kollár, J.: Continuous linear combinations of polynomials. In From Fourier analysis and number theory to Radon transforms and geometry, 233-282. Dev. Math. 28, Springer, New York, 2013.

[14] Glaeser, G.: Étude de quelques algèbres tayloriennes. J. Analyse Math. 6 (1958), 1-124; erratum, insert to 6 (1958), no. 2.

[15] Kollár, J.: Continuous closure of sheaves. Michigan Math. J. 61 (2012), no. 3, 475-491.

[16] Shvartsman, P.: Lipschitz sections of set-valued mappings and traces of functions from the Zygmund class on an arbitrary compactum. Dokl. Akad. Nauk SSSR 276 (1984), no. 3, 559-562.

[17] Shvartsman, P.: On Lipschitz selections of affine-set valued mappings. Geom. Funct. Anal. 11 (2001), no. 4, 840-868.

[18] Shvartsman, P.: Barycentric selectors and a Steiner-type point of a convex body in a Banach space. J. Funct. Anal. 210 (2004), no. 1, 1-42.

[19] Shvartsman, P.: The Whitney extension problem and Lipschitz selections of set-valued mappings in jet-spaces. Trans. Amer. Math. Soc. 360 (2008), no. 10, $5529-5550$.

[20] Whitney, H.: Differentiable functions defined in closed sets. I. Trans. Amer. Math. Soc. 36, no. 2, 369-387.

[21] Whitney, H.: Functions differentiable on the boundaries of regions. Ann. of Math. (2) 35 (1934), no. 3, 482-485.

Received September 10, 2012.

Charles Fefferman: Department of Mathematics, Princeton University, Fine Hall, Washington Road, Princeton, NJ 08544, USA.

E-mail: cf@math.princeton.edu

Garving K. Luli: Department of Mathematics, UC Davis, One Shields Avenue, Davis, CA 95616, USA.

E-mail: kluli@math.ucdavis.edu 